BMJ Open

Sport \&

Exercise

Medicine

\title{
Clinical evaluation of education relating to nutrition and skeletal loading in competitive male road cyclists at risk of relative energy deficiency in sports (RED-S): 6-month randomised controlled trial
}

\author{
Nicola Keay, ${ }^{\oplus 1}$ Gavin Francis, ${ }^{2}$ Ian Entwistle, ${ }^{1}$ Karen Hind ${ }^{\circledR 3}$
}

To cite: Keay N, Francis G, Entwistle I, et al. Clinical evaluation of education relating to nutrition and skeletal loading in competitive male road cyclists at risk of relative energy deficiency in sports (RED-S): 6-month randomised controlled trial. BMJ Open Sport \& Exercise Medicine 2019;5:e000523. doi:10.1136/ bmjsem-2019-000523

- Additional material is published online only. To view please visit the journal online (http://dx.doi.org/10.1136/ bmjsem-2019-000523).

Accepted 5 March 2019

Check for updates

\section{(C) Author(s) (or their} employer(s)) 2019. Re-use permitted under CC BY-NC. No commercial re-use. See rights and permissions. Published by BMJ.

${ }^{1}$ Department of Sport and Exercise Sciences, Durham University, Durham, UK

${ }^{2}$ Science4Performance, London, UK

${ }^{3}$ Department of Sport and Exercise Sciences and the Wolfson Institute for Health and Wellbeing, Durham University, Durham, UK

\section{Correspondence to} Dr Nicola Keay; nickykeayfrancis@googlemail. com

\section{ABSTRACT}

Objective To clinically evaluate education to improve eating behaviour and skeletal loading exercise in male cyclists at risk of poor bone health and impaired performance due to relative energy deficiency in sports. Methods Early race season, 50 competitive male road cyclists were matched, in pairs, based on Z-scores for lumbar spine bone mineral density (BMD). One member of each pair was randomly allocated to receive educational interventions. After the season, 45 cyclists returned for dual-energy $\mathrm{X}$-ray absorptiometry scans and blood tests. Least significant change was applied to identify clinically meaningful BMD changes. Cyclists completed a followup sport-specific questionnaire and clinical interview to ascertain adherence to the interventions.

Results The questionnaire and clinical interview categorised behaviour changes as positive, negative or unchanged. Positive changes in nutrition and skeletal loading were associated with a statistically significant increase of $2.0 \%$ in lumbar spine BMD; 7 of 11 cyclists increases were clinically meaningful. Negative changes in both behaviours were associated with a significant decrease of $2.7 \%$ in lumbar BMD; all nine cyclists' BMD decreases were clinically meaningful. Regarding performance, taking account of functional threshold power, changes in nutritional behaviour accounted for gains or losses of 95 British Cycling racing points. Cyclists reported psychological barriers to change in behaviours, specifically fear of negatively impacting performance.

Conclusions Educational nutritional and skeletal loading interventions can improve bone health, well-being and race performance in male cyclists over a 6-month race season. Psychological support may be required to help some athletes change behaviour.

\section{INTRODUCTION}

Competitive road cyclists are at risk of developing the health and performance consequences of low energy avaiability described in the relative energy deficiency in sport (RED-S) clinical model. ${ }^{1}$ Low energy
What are the key new findings of this study?

Changing both nutrition and skeletal loading exercises over a race season resulted in significant changes in lumbar spine bone mineral density.

- Based on British Cycling points won over the season, reducing energy availability was associated with negative cycling performance; increasing energy availability was associated with superior cycling performance.

- For cyclists to change their behaviours to achieve better health and performance, psychological barriers (fear of performance decrements) were a greater impediment than physical factors (eg time).

How might this study impact clinical practice in the future?

Athletes at risk of relative energy deficiency in sports (RED-S), including male athletes, should be assessed for low energy availability.

- Our sport-specific energy availability questionnaire combined with clinical interview (SEAQ-I) provides a practical, effective clinical tool to identify and manage cyclists at risk of RED-S.

- We suggest the approaches in this study can be applied more broadly to provide sports-specific/ dance-specific educational support on nutritional and exercise strategies to improve health and performance.

- Our findings will be made available to athletes, coaches, parents and healthcare professionals through educational online resources on RED-S, such as the British Association of Sport and Exercise Medicine, www.Health4Performance.co.uk and via the TrainBrave campaign to raise awareness of RED-S.

availability can arise unintentionally and/ or intentionally from restrictive nutrition, as cycling is a gravitational sport where low body weight confers a performance advantage, up 
to a point, until cumulative low energy availability leads to adverse clinical outcomes of RED-S. ${ }^{23}$

The process of bone formation is sensitive to low energy availability. ${ }^{4}$ Lacking the early warning sign of stress fracture experienced by runners, ${ }^{5}$ impaired bone health in cyclists, as a consequence of low energy availability and lack of osteogenic stimulus, can become severe. ${ }^{3}$ Poor bone health has short-term and long-term consequences, with fracture being the most frequent type of injury reported in cyclists. ${ }^{6}$

Early identification of cyclists at risk of developing low energy availability is important in order to intervene to prevent the health and performance consequences of RED-S. As reported in our study of 50 male competitive road cyclists, a Sport-specific Energy Availability Questionnaire and Interview (SEAQ-I) was effective in identifying a low Z-score of lumbar spine bone mineral density (BMD), ${ }^{3}$ which is recognised as a quantifiable measure of low energy availability as outlined in RED-S Clinical Assessment Tool. ${ }^{7}$

However, once male cyclists at risk of RED-S have been identified, there are currently no clear guidelines on how to effectively manage such athletes. ${ }^{2}$ Therefore the purpose of this study was to investigate the efficacy of an educational intervention, specific to competitive cyclists, to ensure adequate energy availability, bone health and performance over the duration of a race season.

\section{MATERIALS AND METHODS Participants}

Forty-five adult male competitive cyclists (equivalent to British Cycling [BC] category 2 or above) who had participated in data collection early in the road race season $^{3}$ returned at the end of the racing season during September and October.

\section{Allocation of cyclists to intervention/no intervention groups}

In the early race season, cyclists were matched, in pairs, based on Z-scores for lumbar spine BMD. One member of each pair was randomly allocated to receive educational interventions from the lead clinician. Technicians performing the dual-energy X-ray absorptiometry (DXA) scans were blinded to the allocation.

\section{Educational nutrition and skeletal loading intervention}

The nutritional advice (see online supplementary file 3) aimed to maintain adequate energy availability, with recommendations for general nutrition and fuelling around training sessions. These recommendations had been approved by registered clinical sports dietitians. The skeletal loading exercises (see online supplementary file 2) were designed to be practical, based on current recommendations to improve bone health, ${ }^{8}$ with input from qualified Pilates teachers and physiotherapists working with cyclists. For cyclists in the intervention group, both the nutrition and exercise recommendations were fully explained and written sheets provided. Online resources included the sheets and a video demonstrating the exercises. Initially weekly, then monthly, these cyclists were contacted to check on progress. All cyclists were asked to record any sustained changes in off-bike exercise and nutrition, both baseline and fuelling around training.

Sport-specific Energy Availability Questionnaire and Interview The clinical assessment of energy availability by SEAQ-I made in our published baseline early race season study $^{3}$ was significantly associated with lumbar spine BMD Z-score in this group of cyclists. We used a similar approach to devise a follow-up SEAQ-I with input from the same clinical sports endocrinologist, sports research scientist, registered clinical sports dietitians, cyclists and coaches for validation of content (see online supplementary file 1 ).

The follow-up SEAQ-I was conducted at the end of the race season. After the cyclists had completed the questionnaire, the sports clinician interviewed them individually to verify answers and gather more details on the responses provided, including the following:

- Cycling training, race results and quantification of any changes in skeletal loading exercises performed, as per recommendations, or otherwise.

- Nutritional information: any changes in baseline nutrition, fuelling around training, as per recommendations, or otherwise; intention to change body weight/composition; addition of any supplements.

- Medical history during study period: number, nature and site of injuries; number of days off training due to illness.

From the follow-up SEAQ-I, cyclists were assessed in each of the areas of nutrition and skeletal loading as implementing either positive change, no change or negative change. Qualitative information was gathered on reasons for cyclists being unable to adhere to the recommendations for their allocated group.

\section{Sport-specific performance measures}

Cyclists recorded the number of $\mathrm{BC}$ race points won during the study period, together with any race highlights. The number of points won was verified from the $\mathrm{BC}$ website. For those competing outside of $\mathrm{BC}$ races (eg, Commonwealth Games, European Championships and time trial events), an equivalent number of $\mathrm{BC}$ points was estimated, to reflect race performance over the season. Cyclists also reported their 60 minute functional threshold power (FTP) in watts.

\section{Bone health measures}

Body weight was measured to the nearest $0.1 \mathrm{~kg}$ using calibrated electronic scales (Seca Alpha, Birmingham UK), and standing height was measured to the nearest $0.1 \mathrm{~cm}$ using a stadiometer (Seca Alpha) with head in Frankfurt plane. Bone health and body composition were evaluated using DXA (GE Lunar iDXA, GE Healthcare, UK) according to best practice recommendations for densitometry in athletes, and the same trained, International 
Society for Clinical Densitometry (ISCD)-certified densitometrist interpreted all examinations to ensure consistency of region of interest placement between baseline and follow-up measures. ${ }^{9}$ BMD was evaluated at the anterior-posterior lumbar spine (L1-L4) and femoral neck. Age-matched BMD Z-scores were derived for each cyclist, at each skeletal site by the DXA software, using UK reference population data (GE Lunar Encore V.15.0, GE Healthcare, Madison, Wisconsin). Precision estimates (coefficient of variation) are $0.4 \%$ for lumbar spine BMD and $0.9 \%$ for femoral neck BMD. ${ }^{10}$ Body composition was derived from a total body scan with precision estimates being $0.5 \%-0.9 \% .^{11}$

\section{Endocrine health measures}

Endocrine and metabolic markers were assessed from capillary blood samples taken in the morning after waking to minimise diurnal variation. Samples were analysed to determine concentrations of total testosterone, vitamin D (25-hydroxy), free triiodothyronine, albumin, calcium, corrected calcium and alkaline phosphatase at Surrey University-accredited laboratories using cobas 8000 analyser with interassay coefficient of variation from $<2 \%$ to $7 \%$ for the markers above. Absolute mean values with SD were determined and results were also expressed as Z-scores, using population mean and SD derived from the definition of the reference range, as covering $95 \%$ of a normal distribution.

\section{Statistical analysis}

Analyses were performed using open source packages Orange $^{12}$ (Bioinformatics Lab at the University of Ljubljana, Slovenia) and SciPy (Enthought, Austin, Texas, USA). The data set included categorical and continuous observations, taken from the follow-up SEAQ-I, blood markers and DXA results. The means and SD of continuous variables were evaluated and, where relevant, compared against appropriate population reference ranges.

Explanatory analyses identified attributes associated with target variables relating to changes in bone health and to cycling performance over the racing season. Since experimental interventions were designed to benefit lumbar spine, the change in lumbar BMD was the target variable for bone health. The target variable for cycling performance was the number of BC points attained.

In accordance with the ISCD recommendations, ${ }^{13}$ the precision error of DXA measurements was taken into account in assessing changes in BMD. A change in $\mathrm{BMD}$ of an individual was considered to be meaningful if exceeding the least significant change (LSC), defined as 2.77 times precision error. ${ }^{9}$ Thus the LSC for lumbar spine BMD was $1.1 \%$.

Changes were considered only for those of the original 50 participants who attended the second round of DXA scans and provided a second set of blood results. The resulting set of paired samples was analysed using the paired sample t-test, after checking for normality
Table 1 Descriptive characteristics of cyclists

\begin{tabular}{lll}
\hline $\mathbf{N}=\mathbf{4 5}$ & Mean \pm SD & $\begin{array}{l}\text { Range: minimum- } \\
\text { maximum }\end{array}$ \\
\hline Age (years) & $36.2 \pm 14.3$ & 18.5 to 72.0 \\
\hline Height $(\mathrm{m})$ & $1.80 \pm 0.06$ & 1.70 to 1.91 \\
\hline Weight $(\mathrm{kg})$ & $73.2 \pm 6.6$ & 62.1 to 91.2 \\
\hline Body mass index $\left(\mathrm{kg} / \mathrm{m}^{2}\right)$ & $22.5 \pm 1.5$ & 18.0 to 25.4 \\
\hline $\begin{array}{l}\text { Training load: average hours } \\
\text { on bike/week }\end{array}$ & $11.2 \pm 4.0$ & 5.0 to 20.0 \\
\hline 60 minute FTP (watts) & $327 \pm 48$ & 195 to 410 \\
\hline FTP/kg (watts/kg) & $4.5 \pm 0.6$ & 2.8 to 5.5 \\
\hline $\begin{array}{l}\text { Race results (equivalent BC } \\
\text { points) }\end{array}$ & $194 \pm 239$ & 0.0 to 953 \\
Number of injuries & $0.4 \pm 0.7$ & 0.0 to 3.0 \\
\hline Number of days of illness & $1.4 \pm 2.5$ & 0.0 to 10.0 \\
\hline
\end{tabular}

BC, British Cycling; FTP, functional threshold power.

using the D'Agostino and Pearson test. The equivalence of means of multiple subgroups was tested by analysis of variance. The significance of the regression coefficients between continuous variables was based on the t-statistic.

\section{Patient and public involvement}

The research, clinical and support pathways for male athletes at risk of RED-S are lacking compared with provision for female athletes. During a pilot study of cyclists, these issues were discussed as SEAQ-I was trialled and refined. Male cyclists were instrumental in the initiation of this current study through involvement of coaches, and recruitment of team-mates and riders from other teams. A male cyclist in this study diagnosed with osteoporosis due to RED-S wrote a patient voice piece for British Journal of Sports Medicine. Other cyclists from the study experiencing consequences of RED-S contributed to articles in cycling magazines and to the TrainBrave campaign to raise awareness. Cyclists and coaches are supportive of and providing input for an educational website on RED-S (www.Health4Performance.co.uk) backed by the British Association of Sport and Exercise Medicine, which has been developed by the authors of this study.

\section{RESULTS}

Of the original 50 cyclists, 45 participants returned for follow-up. Descriptive characteristics are shown in table 1.

\section{Follow-up SEAQ-I and definition of groups}

Although the study design randomly allocated half the athletes to receive educational recommendations, it became apparent during the follow-up SEAQ-I that participants from both groups had followed nutritional and exercise behaviours that deviated from their allocation in the research protocol. Based on the clinical assessment from the follow-up SEAQ-I, athletes were classified in terms of their nutrition and exercise behaviours. 
From the follow-up SEAQ-I, the resultant groupings for changes in energy availability were classified as negative $(n=11)$, no change $(n=22)$ or positive $(n=12)$, and for skeletal loading exercise as negative $(n=12)$, no change $(n=16)$ or positive $(n=17)$. A positive correlation was observed between the nutrition and exercise behaviours $(\mathrm{r}=0.78)$, with change or no change in these behaviours tending to occur together.

Psychological factors were important in certain subgroups. The 11 cyclists who made positive changes in both behaviours reported improved well-being and feeling stronger on the bike. The 13 cyclists who made no behavioural changes included 3 riders, initially in chronic low energy availability, who had been allocated to intervention. These three were unable to sustain the protocol, citing psychological rather than physical barriers, such as stress during the race season and difficulty deviating from their established training and nutrition schedules.

No cyclists had been advised to reduce either nutrition intake or reduce off-bike exercise. Nevertheless, nine riders were assessed as having reduced both. The clinical interview revealed that these behaviours were pursued in the belief that performance would improve. However, these cyclists reported fatigue, illness and injury.

\section{Bone health and body composition}

For the study group as a whole, BMD was lowest at the lumbar spine (Z-score -0.91). Behavioural group was the factor most closely associated with change in BMD at this skeletal site. Table 2 shows the mean change in lumbar BMD according to the subgroups defined in terms of change in energy availability or skeletal loading. Shown in the bottom row of this matrix, cyclists who had reduced energy availability saw a significant average $2.3 \%$ reduction in lumbar BMD over the 6-month interval between scans, compared with a significant $2.2 \%$ increase in lumbar BMD for those who had improved energy availability and little change for those with consistent energy availability. The difference between the energy availability groups was highly significant $(\mathrm{p}<0.001)$. In the far right column of the matrix, cyclists who had increased skeletal loading saw a significant average $1.4 \%$ increase in lumbar BMD, compared with a significant $2.5 \%$ decrease in lumbar BMD for those who had reduced skeletal loading and no change for those maintaining pre-existing exercise regimens. The difference between skeletal loading groups was highly significant $(\mathrm{p}<0.001)$.

Although the association between changes in lumbar BMD with changes in energy availability was slightly higher than with changes in skeletal loading, it was difficult to distinguish between the two factors due to the high correlation between the subgroups. Nevertheless, an increase in both energy availability and skeletal loading led to a markedly better outcome than doing the opposite $(\mathrm{p}<0.001)$.

Figure 1 shows the change in lumbar spine BMD for the individual cyclists, grouped according to changes in behaviours. The colours refer to nutrition and the shapes signify skeletal loading. Significant increases in BMD (exceeding LSC) were found in 7 of the 11 cyclists implementing positive changes in both behaviours (green triangles). Conversely, all nine in the group who implemented negative changes in both behaviours (red crosses) showed significant reductions in BMD. In the remainder of the cyclists, three of four who had positively changed one of the behaviours showed significantly increased BMD, whereas four of six who had negatively changed one of the behaviours exhibited significantly decreased BMD.

\section{Endocrine and metabolic biomarkers}

Endocrine and metabolic biomarkers are found in table 3. A significant increase was seen in vitamin D concentration. Although this could have been due to exposure to sunshine over the summer, the increase was better explained statistically by the increase in the number of cyclists taking sports informed vitamin D supplement of $1000 \mathrm{IU}$ per day, recommended to participants who were not already doing so, based on the low readings in the early season analysis. A significant increase in free triiodothyronine was found. The mean concentration of testosterone remained in the lower half of the reference range. No meaningful, statistically significant relationships were observed between changes in biomarkers and the energy availability and skeletal loading behavioural subgroups.

\section{Determinants of cycling performance}

One cyclist had been signed for a world tour team, three cyclists gained an elite racing licence, and four riders

Table 2 Percentage changes in lumbar BMD by behavioural group

\begin{tabular}{lllllr} 
& & \multicolumn{2}{l}{ Energy availability assessment } & \multirow{2}{*}{ Total } \\
\cline { 3 - 5 } & & Negative & No change & Positive & $2.0(11)$ \\
\hline Skeletal & Positive & NA (0) & $0.3(6)$ & $4.9(1)$ & $0.0(16)$ \\
Loading & No change & $-0.7(2)$ & $-0.3(13)$ & NA (0) & $-2.5(12)$ \\
Exercise & Negative & $-2.7(9)$ & $-1.8(3)$ & $2.2(12)$ & $0.0(45)$ \\
\hline & Total & $-2.3(11)$ & $-0.4(22)$ &
\end{tabular}

Percentage change in L1-L4 lumbar BMD (number of observations).

Significant differences were observed between the groups (see text).

BMD, bone mineral density; NA, not available. 


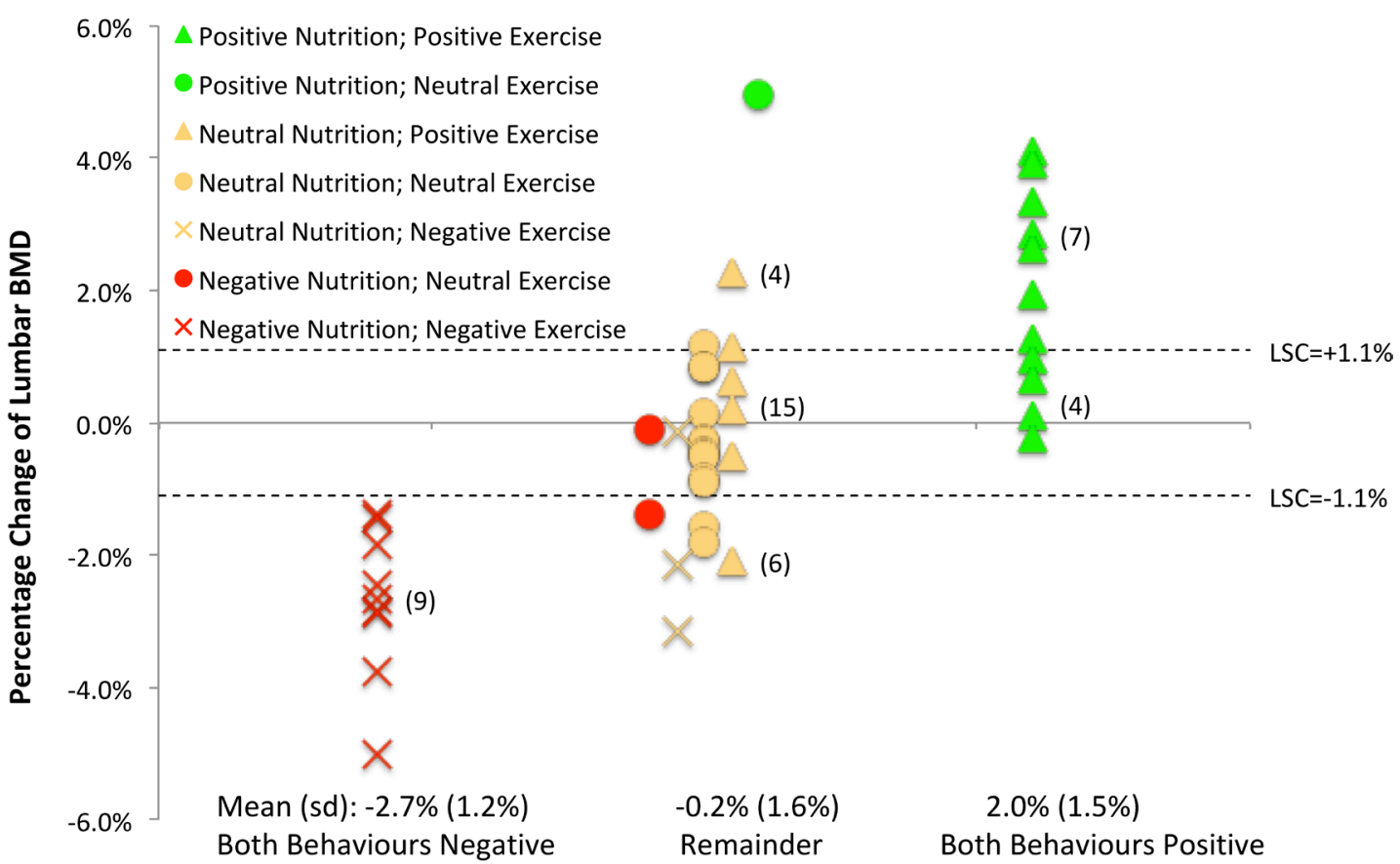

Figure 1 Change in BMD for individual cyclists according to behaviours. BMD, bone mineral density; LSC, least significant change.

moved up from the second to the first BC category. Two riders had stopped racing, while the remainder retained their race category.

The factors most closely associated with performance, assessed by $\mathrm{BC}$ points gained over the racing season, were measures of power (FTP and FTP/kg) and training (weekly training load). Figure 2 shows a significant positive correlation $(\mathrm{p}<0.001)$ between $\mathrm{BC}$ points and 60 minute FTP. The green triangles are cyclists who improved their energy availability, the red crosses represent those who reduced their energy availability, and the blue circles did not change their energy availability over the period.

The majority of those with improved energy availability lay above the line of best fit, whereas most of those with reduced energy availability fell below. A simple two-factor ordinary least squares model of BC points displayed significant t-statistics on the regression coefficients of FTP (3.49, $\mathrm{p}<0.001)$ and energy availability (95.0, $\mathrm{p}=0.015)$. This suggested that improving energy availability was worth $95 \mathrm{BC}$ points, whereas restricting nutrition cost 95 BC points.

Table 3 Endocrine and metabolic biomarkers

\begin{tabular}{|c|c|c|c|c|}
\hline Biomarker & Mean \pm SD & $\begin{array}{l}\text { Range: minimum to } \\
\text { maximum }\end{array}$ & Change in mean & $\begin{array}{l}P \text { value where } \\
\text { significant }\end{array}$ \\
\hline Albumin (35 to $52 \mathrm{~g} / \mathrm{L})$ & $44.9 \pm 3.0$ & 36.8 to 51.0 & 1.62 & 0.001 \\
\hline Albumin, Z-score & $0.36 \pm 0.73$ & -1.54 to 2.06 & 0.42 & \\
\hline Alkaline phosphatase (0 to 129/149 IU/L) & $64.4 \pm 23.5$ & 33.0 to 156.0 & -0.62 & \\
\hline Alkaline phosphatase, Z-score & $-0.03 \pm 0.62$ & -0.96 to 2.14 & -0.04 & \\
\hline Corrected calcium (2.12 to $2.5 \mathrm{mmol} / \mathrm{L}$ ) & $2.40 \pm 0.11$ & 2.17 to 2.78 & -0.02 & \\
\hline Corrected calcium, Z-score & $0.94 \pm 1.15$ & -1.44 to 4.85 & -0.06 & \\
\hline Testosterone (total) $(6.68 / 8.64$ to $25.7 / 29.0 \mathrm{nmol} / \mathrm{L})$ & $14.88 \pm 4.18$ & 8.15 to 23.10 & -0.04 & \\
\hline Testosterone (total), Z-score & $-0.66 \pm 0.88$ & -2.05 to 1.26 & -0.01 & \\
\hline Triiodothyronine (free) (3.1 to $6.8 \mathrm{pmol} / \mathrm{L}$ ) & $5.29 \pm 0.71$ & 3.83 to 6.95 & 0.48 & $<0.001$ \\
\hline Triiodothyronine (free), Z-score & $0.37 \pm 0.76$ & -1.19 to 2.12 & 0.50 & \\
\hline Vitamin D (25-hydroxy) (50 to $175 \mathrm{nmol} / \mathrm{L})$ & $90.6 \pm 23.8$ & 57.3 to 143.0 & 13.0 & 0.001 \\
\hline Vitamin D (25-hydroxy), Z-score & $-0.69 \pm 0.75$ & -1.73 to 0.96 & 0.41 & \\
\hline
\end{tabular}

Values in parentheses indicate relevant population reference ranges (age-specific for testosterone).

Z-scores calculated from reference range (age-specific for testosterone).

The change in mean is for all 45 cyclists post season versus pre-season. 


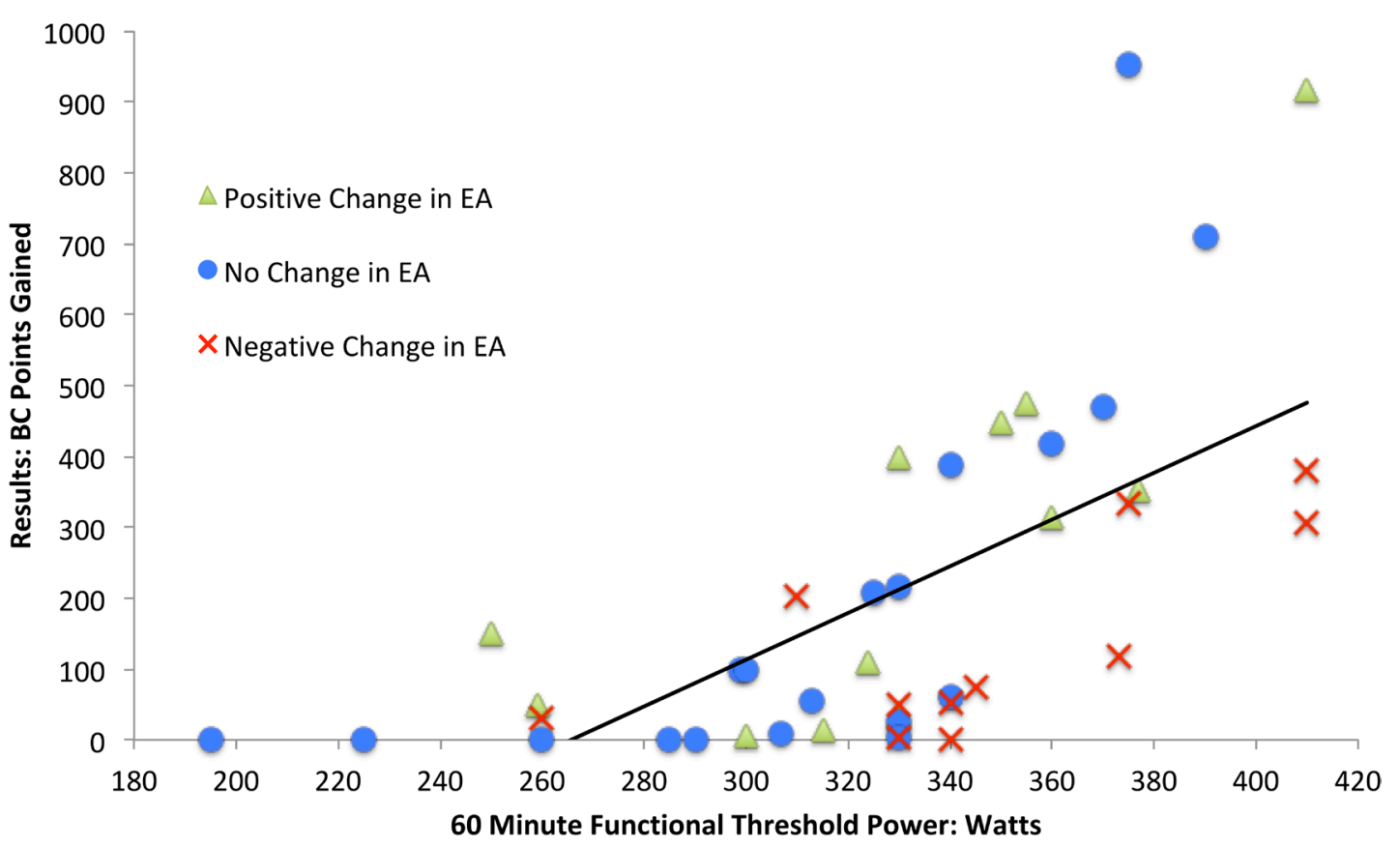

Figure 2 Graph of cycle performance. BC, British Cycling; EA, energy availability.

\section{DISCUSSION}

Over a 6-month race season, positive, cycling-specific, nutritional and skeletal loading improved bone health and performance of competitive male road cyclists at risk of RED-S. Conversely negative changes in energy availability and skeletal loading behaviours corresponded with clinically significant reductions in both bone health and cycling performance. Fear of negatively impacting performance may create a barrier to implementing positive behavioural changes.

\section{The energy availability questionnaire and interview (SEAQ-I) and subgroups}

The follow-up SEAQ-I revealed that psychological, rather than physical, challenges prevented cyclists from adhering to the recommendations for their allocated group. This demonstrates the importance of including a clinical interview when assessing athletes at risk of RED-S. ${ }^{2}$ The correlation between the nutritional and exercise behaviours aligns with athlete psychology. The rigidity in these behaviours could reflect the interactive effect of exercise compulsion, monitoring training metrics and disordered eating. ${ }^{14}$

\section{Effects of positive changes in energy availability and skeletal loading exercise}

A clinically significant increase in lumbar spine BMD was found in cyclists who implemented the recommended strategies. This underlines the priority of addressing low energy availability in RED-S ${ }^{2}$ and concurs with superior bone health reported in cyclists performing off-bike resistance training. ${ }^{15}$ The novel finding in this study is that the combination of cycling-specific, practical, integrated nutritional and skeletal loading measures is effective over a race season in improving the bone health of cyclists.
The synergistic effect of improved energy availability and skeletal loading on bone health in cyclists could be the result of increased energy availability in general to mitigate risk of chronic low energy availability from high training volume undertaken in common with some sports ${ }^{16}$ and compounded in road cycling being weight-sensitive. Furthermore, the recommendation to fuel adequately during long training sessions, characteristic of cycling, would mitigate risk of cumulative acute low energy availability. Our recommendation that cyclists refuel rapidly with a milk-based shake is consistent with studies where pretraining calcium-rich intake inhibited bone resorption after exercise in male ${ }^{17}$ and female ${ }^{18}$ cyclists, and similarly postexercise intake of protein and carbohydrate had positive effects on bone turnover in male athletes. ${ }^{19}$ In the current study, the increase in vitamin D levels would likely contribute to suppression of parathyroid hormone and therefore reduce bone resorption. Vitamin D also promotes muscle strength and improves immune function in athletes. ${ }^{20}$

The results demonstrated qualitative improvements in well-being and quantitative benefits in terms of race results. This subjective self-reported well-being in athletes is recognised as being informative in athlete monitoring and reflective of measured metrics of training load. ${ }^{21}$

\section{Effects of reduction in energy availability and skeletal loading exercise}

Cyclists demonstrating negative behaviours in nutrition and skeletal loading experienced a significant decrease in lumbar spine BMD during the study period. This rate of BMD loss is commensurate with that in astronauts performing 2 hours daily of gravity-simulated exercise on the international space station. ${ }^{22}$ 
Intentional restrictive intake in cyclists over a season concurs with another smaller study. ${ }^{23}$ Psychological characteristics of athletes can initiate and maintain disordered eating behaviours that are implicated in both cause and effect of RED-S. ${ }^{2}$

Those cyclists intentionally restricting dietary intake reported the reason was an attempt to reduce body weight for the race season, in the belief that this would improve race results. Nevertheless, this did not have the desired outcome as fatigue, illness and injury (including fracture) were reported. After accounting for FTP, those restricting energy availability accrued 95 fewer BC points over the season. This demonstrates that competitive cyclists reach a point where attempts to become even leaner lead to adverse outcomes in both health and performance.

\section{Effects of no change in energy availability and skeletal loading exercise}

For those cyclists who did not change either nutrition or off-bike exercise, there was no significant change in lumbar spine BMD, and performance results were more closely correlated with FTP.

\section{Limitations}

Although adherence to change and to no change in behaviours was self-reported, discussion at individual clinical interview provided the opportunity to explore the reasons behind any challenges encountered.

\section{CONCLUSIONS}

Cyclists have multiple risk factors for developing RED-S. We found strong statistical evidence showing changes in energy availability and skeletal loading exercise were positively correlated with clinically significant change in lumbar spine BMD over a 6-month cycle race season. Synergist effects of changes in the combination of both activities were reflected in the greatest improvement or deterioration of lumbar BMD. Furthermore, after accounting for FTP, changes in energy availability resulted in \pm 95 BC points over a race season. This study found that fear of negatively impacting performance prevented some cyclists from implementing behavioural changes to address RED-S. It is hoped that these statistical results might help athletes at risk of developing the consequences of low energy availability overcome the psychological barriers to make the behavioural changes required to improve their health and performance.

Acknowledgements Thank you to all the cyclists and coaches for their interest and participation in this study. Many thanks to Sophie Killer and Renne McGregor for comments on sports performance nutrition. Thanks to Jamie Francis for his input on cycling aspects in study design and comments on the manuscript, and to Brian Oldroyd for scanning of cyclists and comments on the manuscript.

Collaborators Brian Oldroyd, Leeds Beckett University, Leeds, UK.

Contributors NK: conceptualisation of the project, development of study design, research funding application, involvement of cyclists, their coaches and sports performance dietitians, conducting clinical sport-specific interviews, drafting and revision of the manuscript. GF: cycling-specific advanced statistical analysis, drafting and revision of the manuscript. KH: development of study design, research funding application, scanning of cyclists and revision of the manuscript. IE: scanning of cyclists and comments on the manuscript.

Funding This study was supported by funding from the British Association of Sport and Exercise Medicine (BASEM) and by SunVit-D3 for the analysis of endocrine and metabolic markers.

Competing interests None declared.

Patient consent for publication Not required.

Ethics approval This study was reviewed and approved by Leeds Beckett University research ethics committee.

Provenance and peer review Not commissioned; externally peer reviewed.

Data sharing statement No unpublished data were used in the preparation of this manuscript.

Open access This is an open access article distributed in accordance with the Creative Commons Attribution Non Commercial (CC BY-NC 4.0) license, which permits others to distribute, remix, adapt, build upon this work non-commercially, and license their derivative works on different terms, provided the original work is properly cited, appropriate credit is given, any changes made indicated, and the use is non-commercial. See: http://creativecommons.org/licenses/by-nc/4.0/

\section{REFERENCES}

1. Mountjoy M, Sundgot-Borgen J, Burke L, et al. The IOC consensus statement: beyond the Female Athlete Triad--Relative Energy Deficiency in Sport (RED-S). Br J Sports Med 2014;48:491-7.

2. Mountjoy M, Sundgot-Borgen JK, Burke LM, et al. IOC consensus statement on relative energy deficiency in sport (RED-S): 2018 update. Br J Sports Med 2018;52:687-97.

3. Keay N, Francis G, Hind K. Low energy availability assessed by a sport-specific Questionnaire and clinical interview indicative of bone health, endocrine profile and cycling performance in competitive male cyclists. BMJ Open Sport Exerc Med 2018;4:e000424.

4. Ihle R, Loucks AB. Dose-response relationships between energy availability and bone turnover in young exercising women. $J$ Bone Miner Res 2004;19:1231-40.

5. Heikura IA, Uusitalo ALT, Stellingwerff T, et al. Low energy availability is difficult to assess but outcomes have large impact on bone injury rates in elite distance athletes. Int J Sport Nutr Exerc Metab 2018;28:403-11.

6. Procyclingstats. Procyclingstats viewed, 2018. Available: https:// www.procyclingstats.com/statistics/start/injuries

7. Sundgot-Borgen J, Burke L, Mountjoy M. Relative energy deficiency in sport (RED-S) clinical assessment tool (cat). Br J Sports Med 2015.

8. Beck BR, Daly RM, Singh MAF, et al. Exercise and sports science Australia (ESSA) position statement on exercise prescription for the prevention and management of osteoporosis. J Sci Med Sport 2017;20:438-45.

9. Hind K, Slater G, Oldroyd B. Interpretation of dual-energy X-ray Absorptiometry-Derived body composition change in athletes: a review and recommendations for best practice. J Clin Densitom 2018.

10. Hind K, Oldroyd B, Truscott JG. In vivo precision of the Ge Lunar iDXA densitometer for the measurement of total-body, lumbar spine, and femoral bone mineral density in adults. $J$ Clin Densitom 2010;13:413-7.

11. Harley JA, Hind K, O'hara JP. Three-compartment body composition changes in elite rugby League players during a super League season, measured by dual-energy X-ray absorptiometry. J Strength Cond Res 2011;25:1024-9.

12. Demsar J, Curk T, Erjavec A, et al. Orange: data mining Toolbox in python. J Mach Learn Res 2013;14:2349-53.

13. Hangartner TN, Warner S, Braillon $P$, et al. The official positions of the International Society for clinical densitometry: acquisition of dual-energy X-ray absorptiometry body composition and considerations regarding analysis and repeatability of measures. Journal of Clinical Densitometry 2013;16:520-36.

14. Plateau CR, Bone S, Lanning $\mathrm{E}$, et al. Monitoring eating and activity: links with disordered eating, compulsive exercise, and general wellbeing among young adults. Int J Eat Disord 2018;51:1270-6.

15. Mathis SL, Caputo JL. Resistance training is associated with higher lumbar spine and hip bone mineral density in competitive male cyclists. J Strength Cond Res 2018;32:274-9. 
16. Sagayama H, Mimura K, Toguchi M, et al. Total energy expenditure in elite open-water swimmers. Applied Physiology, Nutrition and Metabolism 2018:1-3

17. Guillemant J, Accarie C, Peres G, et al. Acute effects of an oral calcium load on markers of bone metabolism during endurance cycling exercise in male athletes. Calcif Tissue Int 2004;74:407-14.

18. Haakonssen EC, Ross ML, Knight EJ, et al. The effects of a CalciumRich pre-exercise meal on biomarkers of calcium homeostasis in competitive female cyclists: a randomised crossover trial. Plos One 2015;10:e0123302.

19. Townsend R, Elliott-Sale KJ, Currell K, et al. The effect of postexercise carbohydrate and protein ingestion on bone metabolism. Med Sci Sports Exerc 2017;49:1209-18.
20. Owens DJ, Allison R, Close GL. Vitamin D and the athlete: current perspectives and new challenges. Sports Med 2018;48(Suppl 1):3-16.

21. Saw AE, Main LC, Gastin PB. Monitoring the athlete training response: subjective self-reported measures Trump commonly used objective measures: a systematic review. $\mathrm{Br} J$ Sports Med 2016;50:281-91.

22. Sibonga JD, Spector ER, Johnston SL, et al. Evaluating bone loss in ISS astronauts. Aerosp Med Hum Perform 2015;86:38-44.

23. Viner RT, Harris M, Berning JR, et al. Energy availability and dietary patterns of adult male and female competitive cyclists with lower than expected bone mineral density. Int J Sport Nutr Exerc Metab 2015;25:594-602. 